\title{
A Stopped-Flow Kinetic Study of the Assembly of Interpolymer Complexes via Hydrogen-Bonding Interactions
}

\author{
Shizhong Luo, ${ }^{\perp}$ Shiyong Liu, ${ }^{*, \perp, \dagger}$ Jian Xu,${ }^{\dagger}$ Hao Liu, ${ }^{\dagger}$ Zhiyuan Zhu, ${ }^{\dagger}$ Ming Jiang,,${ }^{\dagger}$ and \\ Chi $\mathrm{Wu}^{\perp, \S}$
}

Department of Polymer Science and Engineering, University of Science and Technology of China, Hefei, Anhui 230026, China; The Hefei National Laboratory for Physical Sciences at Microscale, Hefei, Anhui 230026, China; Department of Macromolecular Science, Fudan University, Shanghai 200433, China; and Department of Chemistry, The Chinese University of Hong Kong, Shatin, N.T., Hong Kong

Received March 15, 2006; Revised Manuscript Received May 3, 2006

\begin{abstract}
The formation of soluble interpolymer complexes from poly(4-vinylphenol- $g$-styrene) ( $\mathrm{PVPh}-g$ PS) and poly(styrene-co-4-vinylpyridine) (STVPy) via hydrogen-bonding interactions in tetrahydrofuran (THF) was investigated with viscometry, laser light scattering (LLS), and stopped-flow light scattering. Upon mixing PVPh- $g$-PS with STVPy solutions in THF, colloidally stable dispersions are obtained, indicating the formation of core-shell structure with the core consisting of hydrogen-bonded interpolymer complexes between PVPh backbone and STVPy and the shell of PS grafts. Both LLS and viscometry results reveal that the complexation between PVPh- $g$-PS and STVPy proceeds stoichiometrically. Reduced viscosities of PVPh- $g$-PS/STVPy-49 and PVPh-g-PS/STVPy-74 mixed solutions exhibit minima at weight fractions of PVPh- $g$-PS $\sim 0.5$ and 0.6 , respectively. At these compositions the interpolymer complexes micelles have the highest scattering light intensities and average densities, $\langle\rho\rangle$. PVPh- $g$-PS/STVPy-25 forms aggregates with quite loose structures and low aggregation numbers, which have the smallest sizes and $\langle\rho\rangle$, as compared to that of PVPh- $g$-PS/STVPy-49 and PVPh- $g$-PS/ STVPy-74. For PVPh- $g$-PS/STVPy-49 and PVPh- $g$-PS/STVPy-74, the average hydrodynamic radius, $\left\langle R_{\mathrm{h}}\right\rangle$, is almost independent of the weight fractions of PVPh- $g$-PS. The kinetics of the assembly of micellar complexes are then studied with stopped-flow light scattering upon mixing two component solutions in THF. The complexation between PVPy- $g$-PS and STVPy-25 is so fast (complete within 1-2 ms) that no relaxation processes are observed, reflecting the loose structure of the formed micellar complexes. The formation of micellar complexes between PVPh-g-PS and STVPy-49 or STVPy-74 occurs within $\sim 1-2 \mathrm{~s}$; light scattering intensities exhibit an initially rapid increase and then reach a plateau. Typical relaxation curves at intermediate mixing ratios can be well-fitted with double-exponential functions. Two sequential steps can then be resolved in the assembly of micellar complexes, which are assigned to the initial formation of nonequilibrium aggregates and subsequent structural rearrangements into the final stable micellar complexes, respectively. The effects of mixing ratios and the densities of hydrogenbonding interaction groups on the complexation kinetics are also studied.
\end{abstract}

\section{Introduction}

There are vast amounts of literature on stimuli-responsive amphiphilic block copolymer micelles. ${ }^{1-4}$ For a double-hydrophilic block copolymer (DHBC), both blocks are initially watersoluble, the alteration of external conditions such as $\mathrm{pH}$, temperature, or ionic strengths can selectively render one block to be insoluble, leading to reversible or even structurally invertible micellization. ${ }^{5-13}$ Such micelles, due to their nanocompartmentalization, tunable sizes, and high stability, have promising applications in fields such as controlled drug delivery, separation technologies, catalysis, and materials nanofabrication. ${ }^{1-4}$ The driving force for block copolymer micellization is generally attributed to the microphase precipitation of the insoluble block and the affinity of the soluble block to the solvent; the phase separation of the insoluble block is arrested at early stages due to steric stabilization of the well-solvated block. ${ }^{14}$ Taking this perspective, any kind of phase separation occurring in solution can be conceptually employed to fabricate colloidal nanoparticles, possessing a micelle-like core-shell structure. ${ }^{15,16}$

\footnotetext{
University of Science and Technology of China.

${ }^{\perp}$ The Hefei National Laboratory for Physical Sciences at Microscale.

$\doteqdot$ Fudan University.

$\S$ The Chinese University of Hong Kong.

* To whom correspondence should be addressed. E-mail: sliu@ustc.edu.cn.
}

This has indeed been proved to be the case. The selective alteration of the solubility of one block can be alternatively realized via polyelectrolyte complexation, ${ }^{17-28}$ hydrogen-bonded complexation, ${ }^{29-32}$ stereocomplexation, ${ }^{33}$ metal ion complexation, ${ }^{34-37}$ etc. In 1991, Ishizu et al. ${ }^{38}$ studied the properties of blend films casting from a mixed solution of poly(acrylic acid$g$ - $\alpha$-methylstyrene) (PAA- $g$-PMPS) and poly(4-vinylpyridine$g$ - $\alpha$-methylstyrene) (P4VP- $g$-PMPS) in benzene/methanol mixed solvent; the hydrogen-bonded complexation between PAA and P4VP backbones led to the formation of micellar complexes with PMPS shell. Although they have not clearly pointed out this in the article, this should be the first proof-of-concept example of complexation-induced micellization. In 1995, Kataoka et al. ${ }^{17}$ and Kabanov et al. ${ }^{23}$ successfully employed polyelectrolyte complexation to prepare stable micellar complexes. Later on, this area has gradually received general interests ${ }^{17-37}$ due to their more promising applications as compared to that of conventional block copolymer micelles. ${ }^{1-4}$ For example, complexation between DNA polyanions and cationic poly(ethylene oxide)- $b$-polyspermine can be utilized to deliver nucleic acids to target cells. ${ }^{23}$

Most literature in this area concentrates on polyelectrolyte complexation-induced micellization, ${ }^{17-28}$ and there are only a few reports on hydrogen-bonded complexation-induced micellization, ${ }^{29,30}$ in addition to the original work by Ishizu et al. ${ }^{38}$ 
Scheme 1. (a) Chemical Structures of PVPh-g-PS Graft Copolymer and STVPy Random Copolymers; (b) Schematic Illustration of the Formation of Micellar Complexes between PVPh- $g$-PS and STVPy in THF

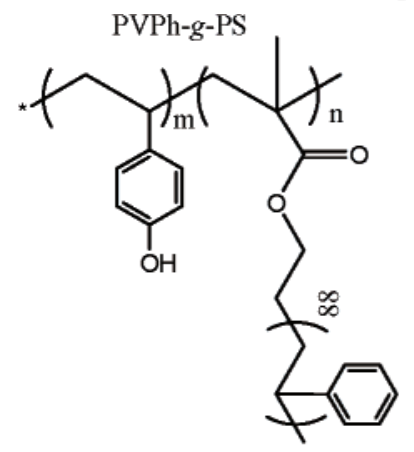<smiles>CC(C)(C)CC(CC(C)(C)C)c1ccncc1</smiles>

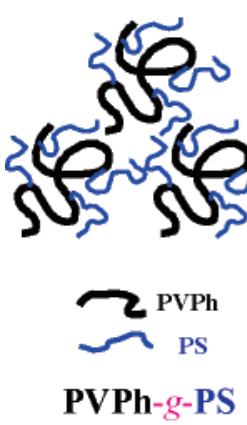

We previously reported the formation of miceller complexes via hydrogen-bonding interactions between poly(styrene- $b$ methyl methacrylate) (PS- $b$-PMMA) and hydroxyl-modified polystyrene, $\mathrm{PS}(\mathrm{OH})$, in toluene. ${ }^{29}$ The formation of core-shell nanoparticles with the core consisting of insoluble PMMA/PS$(\mathrm{OH})$ complexes was evidenced from viscometry, nonradiative energy transfer (NRET) fluorospectroscopy, and laser light scattering (LLS). Topouza et al. ${ }^{30}$ reported the formation of thermoresponsive micellar complexes from poly(styrene- $b-2-$ vinylpyridine) (PS- $b-\mathrm{P} 2 \mathrm{VP}$ ) and poly(methacrylic acid) (PMAA) in dioxane. Hydrogen-bonding interactions between the amine moieties of P2VP block and carboxyl groups of PMAA lead to the formation of micelle-like nanoparticle with a core consisting of the insoluble PMAA/P2VP complexes and a shell of soluble PS block.

However, most of the above literature is about the structural characterization of the final stable micellar complexes; studies of the kinetics of interpolymer complexation ${ }^{39,40}$ and complexation-induced micellization ${ }^{15}$ are really scarce. Morawetz et al. ${ }^{39,40}$ studied the kinetics of hydrogen-bonded interpolymer complexation between dansyl-labeled PAA and PEO. They monitored the complexation kinetics from the evolution of fluorescence intensity with time, which increases upon complexation due to that dansyl groups are now located in a more hydrophobic environment. The kinetic traces are fitted with double-exponential functions, leading to two rate constants. Assignments of these two rate constants to specific physical processes were not given. Moreover, to avoid precipitate formation, they used excess of PEO in relative to that of PAA; this added further complexities. Stuart et al. ${ }^{15}$ reported the kinetics of the formation of micelles with polyelectrolyte complex cores from PAA and poly((dimethylamino)ethyl methacrylate)- $b$-poly(glyceryl methacrylate) (PDMA- $b$-PGMA) in aqueous solution at $\mathrm{pH}$ 7. Upon mixing, they unexpectedly observed the decrease of scattering intensities with time, while we know that the formation of micellar complexes will indeed increases the scattering intensities in relative to that of initially molecularly dissolved solutions. They concluded that the

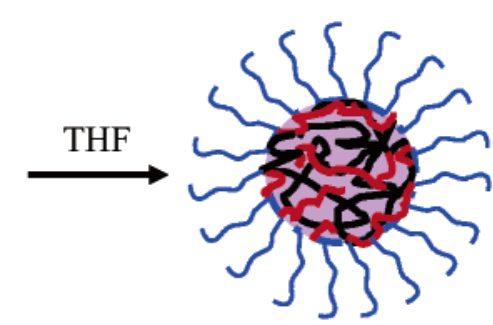

(b)

\section{PVPh- $g$-PS/STVPy complex}

formation of final stable micellar complexes is preceded by a macroscopic phase separation, followed by subsequent rearrangement. ${ }^{15}$

So there is a lack of kinetic studies of the process of interpolymer complexation-induced micellization; more systems need to be investigated before we can draw general conclusions. Understanding the kinetic process will also enable us to control the final structure and properties of the micellar complexes; it is not only of scientific interests but also relevant to their practical applications such as drug delivery and gene transfer. $^{23,41,42}$

Stopped flow is a useful tool for kinetic studies of processes with relaxation times down to $1-2 \mathrm{~ms}$. This technique has been widely used in biophysics, such as protein folding. ${ }^{43}$ Recently our main research interests are concerned with the kinetics of supramolecular self-assembly and single chain folding. We have successfully employed stopped-flow to study the micellization kinetics of DHBCs,${ }^{44}$ the kinetics of the transition from spherical to wormlike micelles, ${ }^{45}$ and single-chain folding kinetics of synthetic polymers. ${ }^{46}$ In the case of interpolymer complexationinduced micellization, the large difference in scattering intensities (mass difference) between the solution containing unimers and complexes makes stopped-flow light scattering technique a convenient and useful apparatus to monitor the kinetic processes.

In this article, we have synthesized poly(4-vinylphenol- $g$ styrene) (PVPh-g-PS) graft copolymer and poly(styrene-co-4vinylpyridine) (STVPy) random copolymers with different 4-vinylpyridine (VPy) contents (25, 49, $74 \mathrm{~mol} \%)$; the chemical structures are shown in Scheme 1. Dai et al. ${ }^{47}$ and Jiang et al. ${ }^{48,49}$ reported that mixing poly(4-vinylphenol) (PVPh) and STVPy solutions in THF led to insoluble hydrogen-bonded interpolymer complexes. In our case, the presence of PS grafts of PVPh-gPS is expected to exert additional steric stabilization to the insoluble complexes, which will prohibit the growth of the complexes coacervate droplets into macroscopic phase separation. A schematic illustration is shown in Scheme 1. Viscometry, dynamic LLs, and static LLS were used to study the soluble 
Table 1. Molecular Parameters of STVPy Copolymers and PVPh- $g$-PS

\begin{tabular}{cccc}
\hline sample code & $\begin{array}{c}\text { VPy or VPh } \\
\text { content }(\mathrm{mol} \%)\end{array}$ & $M_{\mathrm{n}} \times 10^{-4}(\mathrm{~g} / \mathrm{mol})$ & $M_{\mathrm{w}} / M_{\mathrm{n}}$ \\
\hline STVPy-25 & 25.3 & 7.6 & 1.74 \\
STVPy-49 & 48.6 & 3.0 & 1.48 \\
STVPy-74 & 74.1 & 5.7 & 1.65 \\
PVPh- $g$-PS & 42.5 & 5.6 & 1.52
\end{tabular}

micellar complexes formed between PVPh- $g$-PS graft copolymer and STVPy random copolymers via hydrogen-bonding interactions; we then studied the kinetics of the formation of micellar complexes by stopped-flow light scattering.

\section{Experimental Section}

Materials. Styrene, VPy, 4-acetoxystyrene, methacroyl chloride, and ethylene oxide were purified by vacuum distillation in the presence of calcium hydride just prior to use. 2,2'-Azobis(isobutyronitrile) (AIBN) was twice recrystallized from ethanol. Other reagents were used as received without further purification.

Sample Preparation. PS macromonomer was prepared by reacting living polystyryl anions with ethylene oxide and then reacted with newly distilled methacroyl chloride. The functionality of PS macromonomer was $\sim 0.92$ as determined by gel permeation chromatography (GPC) and ${ }^{1} \mathrm{H}$ NMR. The number-average molecular weight $\left(M_{\mathrm{n}}\right)$ of PS macromonomer is $9100 \mathrm{~g} / \mathrm{mol}$ with polydispersity index $\left(M_{\mathrm{w}} / M_{\mathrm{n}}\right)$ of 1.18. Poly(4-vinylphenol- $g$-styrene) ( $\mathrm{PVPh}-\mathrm{g}$-PS) was synthesized by radical copolymerization of 4-acetoxystyrene and PS macromonomer in 1,4-dioxane using AIBN as radical initiator; the polymerization was stopped at $\sim 15 \%$ conversion. The reaction mixture was diluted with THF and precipitated into excess of $n$-hexane; the collected solid was hydrolyzed with hydrazine hydrate in 1,4-dioxane to afford PVPh$g$-PS. ${ }^{50}$ The product was then extracted with cyclohexane and methanol for $12 \mathrm{~h}$ to remove residual PS macromonomer or unfunctionalized PS and possible PVPh homopolymer. $M_{\mathrm{n}}$ of PVPh$g$-PS is determined to be $5.6 \times 10^{4} \mathrm{~g} / \mathrm{mol}$ with $M_{\mathrm{w}} / M_{\mathrm{n}}=1.52$. The molar content of VPh in PVPh-g-PS is $42.5 \mathrm{~mol} \%$. On average, there are $\sim 3.5$ grafted PS chain per graft copolymer chain.

STVPy random copolymers were synthesized by random copolymerization of styrene and 4-vinylpyridine at $60{ }^{\circ} \mathrm{C}$ using AIBN as the initiator. ${ }^{48}$ Polymerizations are typically stopped at $\sim 20 \%$ conversion. The molecular weights and vinylpyridine (VPy) contents of STVPy random copolymers were determined by GPC and ${ }^{1} \mathrm{H}$ NMR. The chemical structures and molecular parameters of PVPy- $g$-PS and STVPy random copolymers are shown in Scheme 1 and Table 1, respectively. The random copolymers were denoted STVPy- $x$, where $x$ represents the VPy molar contents in the copolymers.

Viscometry. Measurements of the reduced viscosities $\eta_{\mathrm{sp}} / C$ of the solution mixture of PVPh- $g$-PS and STVPy in THF were conducted as a function of composition using an Ubbelohde viscometer at $25 \pm 0.05{ }^{\circ} \mathrm{C}$. The original concentrations of the component polymers were kept at $1.5 \times 10^{-3} \mathrm{~g} / \mathrm{mL}$. STVPy samples with VPy contents at three levels, i.e., 25, 49, $74 \mathrm{~mol} \%$, were used. The reduced viscosities were measured $10 \mathrm{~min}$ after thoroughly mixing PVPh- $g$-PS and STVPy solutions in the viscometer.

Laser Light Scattering (LLS). A commercial spectrometer (ALV/DLS/SLS-5022F) equipped with a multitau digital time correlation (ALV5000) and a cylindrical $22 \mathrm{~mW}$ UNIPHASE HeNe laser $\left(\lambda_{0}=632 \mathrm{~nm}\right)$ as the light source was used. The incident beam was vertically polarized with respect to the scattering plane. All LLS measurements were done in THF at $25.0 \pm 0.1{ }^{\circ} \mathrm{C}$. The specific refractive index increment $(\mathrm{d} n / \mathrm{d} C)$ was determined by a novel and precise differential refractometer. ${ }^{51} \mathrm{~d} n / \mathrm{d} C$ values of PVPh- $g$-PS, STVPy-25, STVPy-49, and STVPy-74 in THF at 25 ${ }^{\circ} \mathrm{C}$ are $0.191,0.201,0.197$, and $0.195 \mathrm{~mL} / \mathrm{g}$, respectively. ${ }^{49}$

In static LLS, we can obtain the weight-average molar mass $\left(M_{\mathrm{w}}\right)$ and the $z$-average root-mean-square radius of gyration $\left(\left\langle R_{\mathrm{g}}{ }^{2}\right\rangle^{1 / 2}\right.$ or written as $\left.\left\langle R_{\mathrm{g}}\right\rangle\right)$ of polymer chains in a dilute solution from the angular dependence of the excess absolute scattering intensity, known as the Rayleigh ratio $R_{\mathrm{vv}}(q)$, as

$$
\frac{K C}{R_{\mathrm{vv}}(q)} \approx \frac{1}{M_{\mathrm{w}}}\left(1+\frac{1}{3}\left\langle R_{\mathrm{g}}{ }^{2}\right\rangle q^{2}\right)+2 A_{2} C
$$

where $K=4 \pi^{2} n^{2}(\mathrm{~d} n / \mathrm{d} C)^{2} /\left(N_{\mathrm{A}} \lambda_{0}{ }^{4}\right)$ and $q=\left(4 \pi n / \lambda_{0}\right) \sin (\theta / 2)$ with $N_{\mathrm{A}}, n, \theta$, and $\lambda_{0}$ being the Avogadro number, the solvent refractive index, the scattering angle, and the wavelength of the laser light in a vacuum, respectively, and $A_{2}$ is the second virial coefficient. The polymer concentration $(C)$ is in units of $\mathrm{g} / \mathrm{mL}$. It should be noted that eq 1 is valid only for a homopolymer solution. However, in the current study, the $\mathrm{d} n / \mathrm{d} C$ values of PVPh- $g$-PS and STVPy copolymes are almost identical, so that we can still use eq 1 for all calculations of static LLS results.

In dynamic LLS, the Laplace inversion of each measured intensity-intensity time correlation function $G^{(2)}(q, t)$ in the selfbeating mode can lead to a line-width distribution $G(\Gamma)$. For a pure diffusive relaxation, $\Gamma$ is related to the translational diffusion coefficient $D$ by $\left(\Gamma / q^{2}\right)_{C \rightarrow 0, q \rightarrow 0} \rightarrow D$ or further to the hydrodynamic radius $R_{\mathrm{h}}$ via the Stokes-Einstein equation, $R_{\mathrm{h}}=\left(k_{\mathrm{B}} T / 6 \pi \eta_{0}\right) / D$, where $k_{\mathrm{B}}, T$, and $\eta_{0}$ are the Boltzmann constant, the absolute temperature, and the solvent viscosity, respectively.

All samples were dissolved in a proper amount of THF at $1.0 \times$ $10^{-4} \mathrm{~g} / \mathrm{mL}$ and clarified with a $0.45 \mu \mathrm{m}$ Millipore PTFE filter. The solution mixtures were prepared by adding dropwise a proper amount of the dust-free PVPh- $g$-PS solution to $\sim 2 \mathrm{~mL}$ of previously clarified STVPy solution under gentle agitation. No precipitates formation is observed at all mixing ratios. All the mixed solutions are stable even after standing for more than 3 months. Nevertheless, fresh solution mixtures were used in LLS. The temperature was kept at $25^{\circ} \mathrm{C}$ during all LLS measurements.

Stopped-Flow with Light Scattering Detection. Stopped-flow studies were carried out using a Bio-Logic SFM300/S stoppedflow instrument. ${ }^{52}$ The SFM-3/S is a three-syringe $(10 \mathrm{~mL})$ instrument in which all step-motor-driven syringes (S1, S2, S3) can be operated independently to carry out single or double mixing. The SFM-300/S stopped-flow device is attached to the MOS-250 spectrometer; kinetic data were fitted using the program Biokine (Bio-Logic). For the light scattering detection at a scattering angle of $90^{\circ}$, both the excitation and emission wavelengths were adjusted to $335 \mathrm{~nm}$ with $10 \mathrm{~nm}$ slits. Dynamic trace at each composition is averaged from 15 to 20 successive shots. Using FC-08 or FC-15 flow cells, the typical dead times are 1.1 and $2.6 \mathrm{~ms}$, respectively. Temperature was maintained at $25^{\circ} \mathrm{C}$ by circulating water around the syringe chamber and the observation head.

\section{Results and Discussion}

Viscometry. Interpolymer complexation in solution always accompanies contraction or collapse of the component polymer chains, resulting in a viscosity decrease, turbidity, or even precipitation, depending on the densities of hydrogen-bonding interaction groups on the polymer chains. For example, the complexation between poly(styrene-co-4-vinylphenol) (STVPh) and STVPy has been studied with viscometry. ${ }^{48}$ When the VPh and VPy contents in the component polymers are relatively high, interpolymer complexation lead to precipitation. Reduced viscosities are measured after filtering the precipitates. Strictly speaking, the viscometry results cannot reflect the actual conformational changes of polymer chains due to mass loss. Thus, the measured reduced viscosities can only be considered as apparent values.

PVPh- $g$-PS and three STVPy copolymers with VPy contents being 25, 49, and $74 \mathrm{~mol} \%$, respectively, can be molecularly dissolved in THF. The mixed solutions of PVPh- $g$-PS and STVPy in THF at all mixing ratios are optically transparent and show no sign of macroscopic phase separation. A closer examination reveals that the mixed solutions of PVPh- $g$-PS/ 


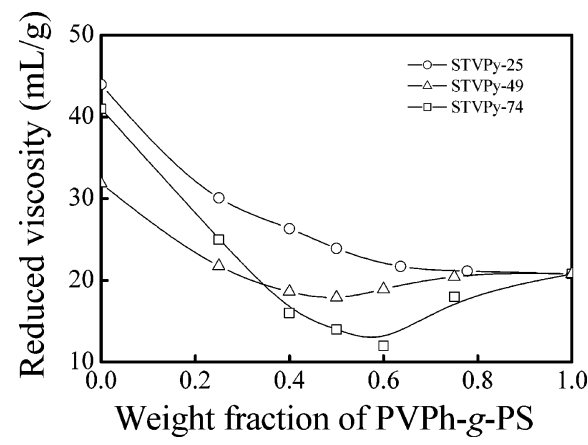

Figure 1. Reduced viscosities of PVPh- $g$-PS/STVPy mixed solutions in THF as a function of the weight fractions of PVPh- $g$-PS. The total polymer concentration is fixed at $1.5 \times 10^{-3} \mathrm{~g} / \mathrm{mL}$.

STVPy-25 are clear, while mixed solutions of PVPh- $g$-PS/ STVPy-49 and PVPh-g-PS/STVPy-74 are slightly bluish, characteristic of colloidal particles of several tens of nanometers.

A comparison between the complexation behavior of STVPh/ STVPy and PVPh- $g$-PS/STVPy reflects the chain architectural effects. ${ }^{48}$ As one component polymer is fixed to be STVPy, the distribution of hydrogen-bonding donor groups (VPh units) on the polymer chain plays an important role. When VPh units are randomly distributed in STVPh copolymers, the complexation between STVPh and STVPy leads to a new insoluble phase; the aggregates between these insoluble droplets grow with time and finally precipitate out. ${ }^{53}$ However, when VPh units are forming a backbone and grafted with PS chains, the phase separation due to complexation between PVPh- $g$-PS and STVPy is stopped at early stages; the insoluble interpolymer complexes form the micellar core and are stabilized by well-solvated PS grafts. This observation is generally in agreement with previous reports. ${ }^{17-30}$ A schematic illustration for the formation of micellar complexes between PVPh- $g$-PS and STVPy in THF is shown in Scheme 1.

Because of the lack of macroscopic phase separation, the interaction between PVPh- $g$-PS and STVPy can be conveniently studied with viscometry. In the viscometry experiment, the total polymer concentration is $1.5 \times 10^{-3} \mathrm{~g} / \mathrm{mL}$, much less than the chain overlapping concentration, $C^{*}$, which can be estimated by $C^{*}=1 /[\eta]$, where $[\eta]$ is the intrinsic viscosity. It is understandable that polymer chains make a much larger contribution to the viscosity in the swollen coil state than in the collapsed aggregates. In the absence of any specific interactions between component polymers, component polymer chains will just act independently; the overall reduced viscosities will just be equal to the additivity value.

Figure 1 shows the variation of the reduced viscosities of PVPh- $g$-PS/STVPy mixed solutions as a function of the weight fraction of PVPh- $g$-PS. All the blend solutions show a negative deviation from the additivity. The negative deviation becomes more substantial when VPy contents of STVPy increase from 25 to $74 \mathrm{~mol} \%$. The negative deviation reveals the interpolymer complexation between PVPh backbone and STVPy copolymers, leading to soluble micellar complexes. ${ }^{29,53}$

Reduced viscosities of PVPh- $g$-PS/STVPy-25 mixed solutions at all ratios do not exhibit any noticeable minimum, while for PVPh-g-PS/STVPy-49 and PVPh- $g$-PS/STVPy-74, viscosity minima are observed at PVPh- $g$-PS weight fractions of $\sim 0.5$ and 0.6 , respectively. The composition at the viscosity minimum should be ascribed to the stoichiometry; ${ }^{52}$ all component polymer chains have participated in the formation of micellar complexes. Indeed, the molar concentrations of VPh and VPy units are quite close at these compositions. Compared to that of PVPh-g-PS/

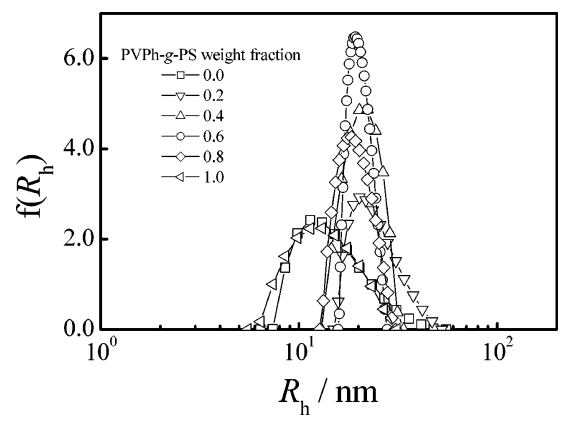

Figure 2. Hydrodynamic radius distributions, $f\left(R_{\mathrm{h}}\right)$, of PVPh- $g$-PS, STVPy-49, and their mixed solutions in THF with different PVPh- $g$ PS weight fractions. The total polymer concentration is $1.0 \times 10^{-4}$ $\mathrm{g} / \mathrm{mL}$. The scattering angle is at $15^{\circ}$.

STVPy-49 and PVPh- $g$-PS/STVPy-74, the reduced viscosities of PVPh- $g$-PS/STVPy-25 mixed solutions only show small negative deviation from the additivity. We have previously reported that $\mathrm{PVPh}$ homopolymer will form insoluble interpolymer complexes in THF upon complexation with STVPy$25 .{ }^{48}$ Hydrogen-bonded interactions between PVPh- $g$-PS and STVPy-25 should have occurred, and the structure of the aggregates is probably very loose. On the contrary, the large deviation of PVPy- $g$-PS/STVPy-49 and PVPh- $g$-PS/STVPy-74 from additivity reflects that the formed micellar complexes are quite compact. LLS results described in the following section will provide more detailed information about this conclusion.

LLS Characterization. The LLS technique is very sensitive to the formation of aggregates, especially in very dilute solutions, where the conventional viscometry technique is not applicable anymore. It should be noted that the LLS technique mainly detects information about aggregates because molecularly dissolved chains contribute little to the scattering intensities. Thus, the LLS technique can be conveniently employed to study the formation of micellar complexes between PVPh- $g$-PS and STVPy.

Figure 2 shows typical hydrodynamic radius distributions, $f\left(R_{\mathrm{h}}\right)$, of PVPh- $g$-PS/STVPy-49 mixed solutions in THF with different weight fractions of PVPh- $g$-PS. Pure PVPh- $g$-PS and STVPy-49 can be molecularly dissolved in THF. The solutions have very low light scattering intensities. The average hydrodynamic radii, $\left\langle R_{\mathrm{h}}\right\rangle$, are $\sim 10 \mathrm{~nm}$, with relatively broad distributions. All the mixed solutions with weight fractions of PVPh$g$-PS ranging from 0.2 to 0.8 are optically transparent and stable, LLS results reveal a shift of the hydrodynamic radius distributions to higher values, with $\left\langle R_{\mathrm{h}}\right\rangle$ of $\sim 20 \mathrm{~nm}$. It can also be clearly seen that the micellar complexes have a relative narrow distribution, with typical polydispersities $\left(\mu_{2} / \Gamma^{2}\right)$ less than 0.1 . The $R_{\mathrm{h}}$ distributions of blend solutions at all mixing ratios are monomodal. It is interesting to note that when the weight fraction of PVPh- $g$-PS is between 0.4 and 0.6 , the formed micellar complexes have the narrowest $R_{\mathrm{h}}$ distributions.

Figure 3 shows the variation of scattering intensities and $\left\langle R_{\mathrm{h}}\right\rangle$ with the weight fraction of PVPh- $g$-PS for mixed solution of PVPh- $g$-PS and STVPy with different VPy contents. From Figure $3 \mathrm{a}$, we can tell that the interaction between PVPh- $g$-PS and STVPy-25 is relatively weak; the scattering intensities of mixed solutions are very low and only a little higher than that of the pure polymer solutions. In combination with the viscometry results, interaction between PVPh- $g$-PS and STVPy-25 should lead to the formation of soluble complex aggregates with quite loose structure and very low aggregation numbers $;{ }^{16}$ it is also highly possible that the formed soluble aggregates do not possess a well-defined core-shell micellar structure. On the 

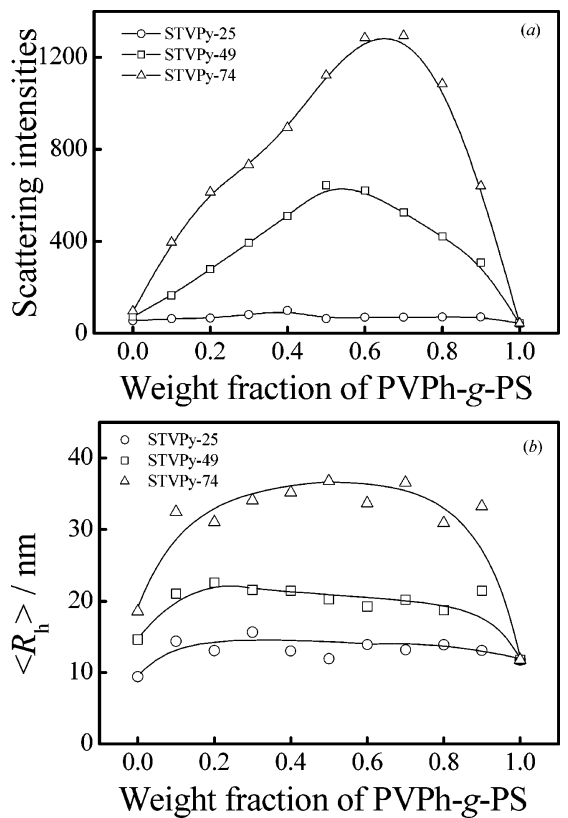

Figure 3. Change of light scattering intensities (a) and the average hydrodynamic radius, $\left\langle R_{\mathrm{h}}\right\rangle$ (b), of PVPh- $g$-PS/STVPy mixed solutions in THF with the weight fractions of PVPh- $g$-PS, where the total polymer concentration is $1.0 \times 10^{-4} \mathrm{~g} / \mathrm{mL}$. The scattering angle is at $15^{\circ}$.

contrary, the mixed solutions of PVPh-g-PS/STVPy-49 and $\mathrm{PVPh}-\mathrm{g}$-PS/STVPy-74 have much higher scattering intensities, exhibiting maxima at PVPh- $g$-PS weight fractions $\sim 0.5$ and 0.6 , respectively. The above observation is also in agreement with viscometry results (Figure 1); reduced viscosities of PVPh- $g$ PS/STVPy-25 mixed solutions have the smallest negative deviation and no noticeable minimum, while that of PVPh- $g$ PS/STVPy-49 and PVPh- $g$-PS/STVPy-74 mixed solution exhibits a minimum at compositions where scattering intensities are the highest.

It can be clearly seen from Figure $3 \mathrm{~b}$ that $\left\langle R_{\mathrm{h}}\right\rangle$ of micellar complexes increases with VPy contents in STVPy. $\left\langle R_{\mathrm{h}}\right\rangle$ are $\sim 14$, 20, and $34 \mathrm{~nm}$ when the VPy contents are 25, 49, and $74 \mathrm{~mol}$ $\%$, respectively. For PVPh- $g$-PS/STVPy-49 and PVPh- $g$-PS/ STVPy-74, $\left\langle R_{\mathrm{h}}\right\rangle$ does not vary a lot with mixing ratios. It is well-known that LLS is very sensitive to interchain aggregates relative to molecularly dissolved chains. The almost constant $\left\langle R_{\mathrm{h}}\right\rangle$ with different mixing ratios reflects that complexation between PVPh- $g$-PS and STVPy proceeds stoichiometrically. The composition of the micellar complexes is independent of the feed ratio, and the micellar complexes coexist with free component polymer chains.

Kataoka et al. ${ }^{18,19}$ have reported the formation of polyion complexes micelles from lysozyme and poly(ethylene glycol)$b$-poly(aspartic acid) (PEG- $b$-PAsp); when lysozyme is in excess, $\left\langle R_{\mathrm{h}}\right\rangle$ remains constant. The stoichiometric micellar complexes coexist with free lysozyme. When PAsp is in excess, they observed the increase of $\left\langle R_{\mathrm{h}}\right\rangle$ with increasing PAsp molar fractions, and they conclude that the formation of micellar complexes in the presence of excess of PAsp may proceed noncooperatively. In the current case, $\left\langle R_{\mathrm{h}}\right\rangle$ does not vary a lot at all mixing ratios, which reflects the stoichiometric complexation between PVPh-g-PS and STVPy. During the polyion complexation, when one component polymer is in excess, there exists the possibility that no micellar complexes are formed; the formed aggregates are very loose, and aggregation into larger, dense objects is retarded by the excess charge of the aggregates. ${ }^{15,16}$ Hydrogen-bonded complexation between PVPh$g$-PS and STVPy should be different. There is no charge
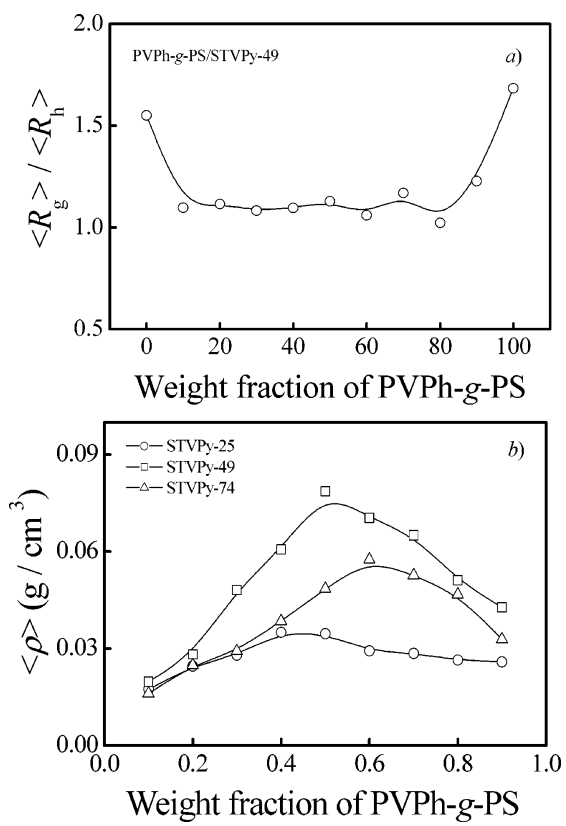

Figure 4. (a) The change of $\left\langle R_{\mathrm{g}}\right\rangle /\left\langle R_{\mathrm{h}}\right\rangle$ for PVPh- $g$-PS/STVPy-49 mixed solution in THF with the weight fractions of PVPh-g-PS. (b) The average densities, $\langle\rho\rangle$, of the micellar complexes in THF as a function of the weight fractions of PVPh- $g$-PS. The total polymer concentration is $1.0 \times 10^{-4} \mathrm{~g} / \mathrm{mL}$.

repulsion between aggregates and component polymer chains; thus, the observed stoichiometric complexation at all mixing ratios seems to be reasonable.

For PVPh- $g$-PS/STVPy-49 mixed solutions, $\left\langle R_{\mathrm{g}}\right\rangle /\left\langle R_{\mathrm{h}}\right\rangle$ is $\sim 1.1$, which keeps almost constant at all mixing ratios (Figure 4a). It is well-known that $\left\langle R_{\mathrm{g}}\right\rangle /\left\langle R_{\mathrm{h}}\right\rangle$ values reflect the conformation of a polymer chain or the density distribution of chain segments in core-shell nanoparticles. ${ }^{54}$ For example, for linear and flexible polymer chains, $\left\langle R_{\mathrm{g}}\right\rangle /\left\langle R_{\mathrm{h}}\right\rangle$ is $\sim 1.5$, but for a uniform nondraining sphere, $\left\langle R_{\mathrm{g}}\right\rangle\left\langle\left\langle R_{\mathrm{h}}\right\rangle\right.$ drops to $0.774 .{ }^{54}$ The almost constant $\left\langle R_{\mathrm{g}}\right\rangle\left\langle\left\langle R_{\mathrm{h}}\right\rangle\right.$ at different mixing ratios further supports that the complexation between PVPh- $g$-PS and STVPy proceeds stoichiometrically.

Figure $4 \mathrm{~b}$ shows the composition dependence of the average densities, $\langle\rho\rangle$, of the micellar complexes from PVPy-g-PS/ STVPy with different VPy contents. Here, $\langle\rho\rangle$ is defined as $\langle\rho\rangle$ $=M_{\mathrm{w}} /\left(\left(N_{\mathrm{A}} 4 \pi\left\langle R_{\mathrm{h}}\right\rangle^{3}\right) / 3\right)$, where $M_{\mathrm{w}}$ can be obtained by static LLS on the basis of eq 1 . The variation of $\langle\rho\rangle$ with the weight fraction of PVPh- $g$-PS in PVPh- $g$-PS/STVPy exhibits a maximum at intermediate mixing ratios, at which the weight fractions of PVPh- $g$-PS are $\sim 0.4,0.5$, and 0.6 when VPy contents are 25, 49 , and $74 \mathrm{~mol} \%$, respectively. This agrees quite well with viscometry (Figure 1) and LLS results (Figure 3).

Figure $4 \mathrm{~b}$ also tells us that $\langle\rho\rangle$ of micellar complexes from PVPh- $g$-PS/STVPy-49 $(\sim 0.078 \mathrm{~g} / \mathrm{mL})$ is relatively larger than that of PVPh- $g$-PS/STVPy-74 $(0.056 \mathrm{~g} / \mathrm{mL})$, while that formed from PVPh- $g$-PS/STVPy-25 has the lowest density; i.e., it will take a quite loose structure.

It should be safe to assume that at composition with the maximum $\langle\rho\rangle$ all component chains have participated in the formation of micellar complexes. $\left\langle R_{\mathrm{h}}\right\rangle$ and $\langle\rho\rangle$ should be the most accurate. $\left\langle R_{\mathrm{h}}\right\rangle$ of micellar complexes from PVPh- $g$-PS/ STVPy-49 and PVPh- $g$-PS/STVPy-74 at compositions with the maximum $\langle\rho\rangle$ is independent of the detecting angle. For spherical particles, the translational diffusion coefficient should be independent of the scattering vector because of the undetectable rotational motion; ${ }^{55}$ thus, micellar complexes formed from 
PVPh- $g$-PS/STVPy-49 and PVPh- $g$-PS/STVPy-74 are most probably spherical in shape.

Stopped-Flow Studies of the Assembly of Micellar Complexes. Through the above analyses, we have successfully obtained the structural information on micellar complexes, together with their dependence on the mixing ratio and densities of interaction groups. We then proceed to use stopped-flow light scattering to study the kinetics of the formation of micellar complexes.

An experiment using a basic stopped-flow apparatus is quite simple in principle. Our Bio-Logic SFM300 stopped-flow uses the drive motor to rapidly fire two solutions, contained in separate drive syringes driven by separate motors, together into a mixing device. The solutions then flow into the observation cell, displacing the previous contents with freshly mixed reactants. Motors cease to push at a given time to limit the volume of solution expended with each experiment and also serve to abruptly stop the flow. In addition, an additional hard stop was used to guarantee the quality of stop. The fresh reactants in the observation cell are illuminated by a light source, and the change, as a function of time, in many optical properties (absorbance, fluorescence, light scattering, turbidity, fluorescence anisotropy, etc.) can be measured. The measurement of these optical properties is performed by the detectors which can be mounted either perpendicular or parallel to the path of incoming light. Regardless of which configuration the stopped flow uses, the time resolution is limited by the time required for the reactants to flow from the final point of mixing to the observation cell. This time is referred to as the dead time of the instrument. The stop of the flow was dually controlled by the three separate motors and the hard stop. Although the mixed solution is subjected to large shear forces before the stop, the effective light scattering detection starts $1-3 \mathrm{~ms}$ (the dead time) after the stop; thus, we assume that shear forces before the stop will not exhibit any appreciable effects on the complexation process. ${ }^{44}$

The kinetics of the assembly into micellar complexes are monitored through the time dependences of scattering intensities at $90^{\circ}$ after stopped-flow mixing component polymer solutions in THF. It is important to note that the scattering light intensities reflect primarily the weight-average molar mass $\left(M_{\mathrm{w}}\right)$ of the micellar particles in the mixed solution. Intensities of light scattered from micellar solutions were at least 2 orders of magnitude larger than those from the corresponding unimer solutions. ${ }^{56}$ On the basis of eq 1 , the slope of the $K C / R_{\mathrm{vv}}(q) \sim$ $q^{2}$ plot is $\left\langle R_{\mathrm{g}}\right\rangle^{2} / 3 M_{\mathrm{w}}$. At a fixed scattering angle of $90^{\circ}$, for micellar complexes with the same $M_{\mathrm{w}}$, those with larger $\left\langle R_{\mathrm{g}}\right\rangle$ will scatter less light than those with smaller $\left\langle R_{\mathrm{g}}\right\rangle$ values. From Figures $3 \mathrm{~b}$ and $4 \mathrm{a}$, we know that $\left\langle R_{\mathrm{g}}\right\rangle$ does not vary a lot with mixing ratios and that $\left\langle R_{\mathrm{g}}\right\rangle$ of micellar complexes is comparable to that of pure PVPh- $g$-PS and PVPy solutions; thus, the effects of micellar size on the scattering intensities detected at $90^{\circ}$ during micellization are neglected, and a comparison of the micellization kinetics at different weight fractions of PVPh- $g$ PS is also reasonable.

Figures 5, 6, and 7 show the time dependence of the scattering light intensities for $\mathrm{PVPh}-g$-PS/STVPy-25, PVPh- $g$-PS/STVPy49, and PVPh-g-PS/STVPy-74 after stopped-flow mixing component polymer solutions in THF. The total polymer concentration is fixed at $1.0 \times 10^{-4} \mathrm{~g} / \mathrm{mL}$. The dynamic traces obtained after stopped-flow mixing PVPh- $g$-PS and STVPy-25 solutions in THF are shown in Figure 5; the scattering intensities remain constant with time at all mixing ratios. From the viscometry and LLS results described earlier, we know the there

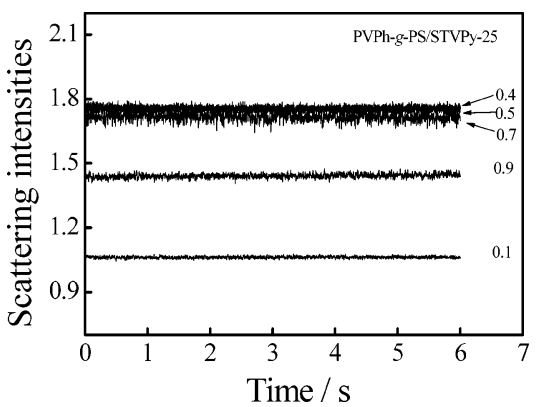

Figure 5. Time dependence of the scattering light intensities upon stopped-flow mixing PVPh- $g$-PS and STVPy-25 solutions in THF at different weight fractions of $\mathrm{PVPh}-\mathrm{g}$-PS; the total polymer concentration after mixing is $1.0 \times 10^{-4} \mathrm{~g} / \mathrm{mL}$.
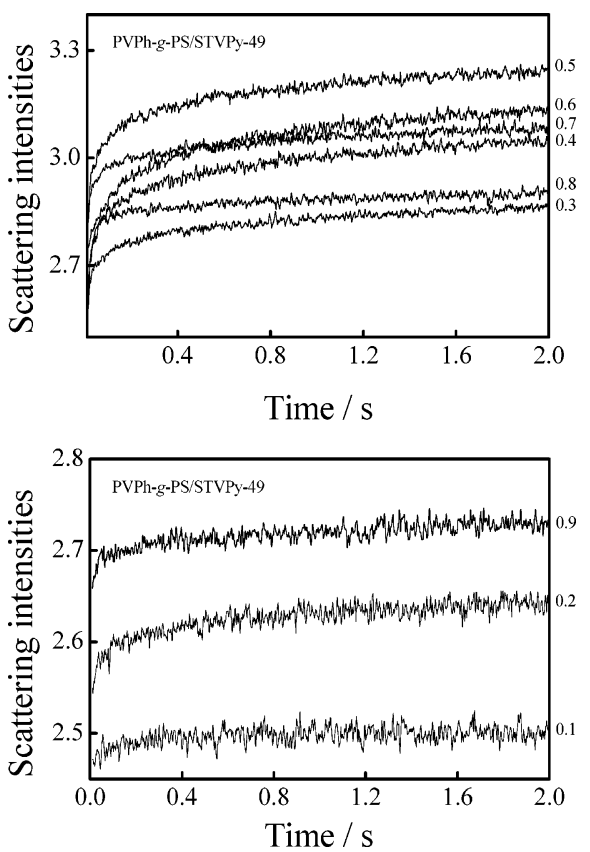

Figure 6. Time dependence of the scattering light intensities upon stopped-flow mixing PVPh- $g$-PS and STVPy-49 solutions in THF at different weight fractions of $\mathrm{PVPh}-g$-PS; the total polymer concentration after mixing is $1.0 \times 10^{-4} \mathrm{~g} / \mathrm{mL}$.

exist interactions (although weak) between PVPh- $g$-PS and STVPy-25; scattering intensities of mixed solutions are a bit higher than that of the pure component polymer solutions. The interaction between PVPh- $g$-PS and STVPy-25 must be so fast that it is completed within the dead time of the stopped-flow $(\sim 1-2 \mathrm{~ms})$. However, we can still tell from Figure 5 that micellar complexes formed at intermediate mixing ratios (weight fractions of PVPh- $g$-PS $\sim 0.4,0.5$, and 0.6) exhibit higher scattering intensities as compared to that at PVPh- $g$-PS weight fractions $\sim 0.1$ and 0.9 ; this is in agreement with LLS results as shown in Figure 3. The fast complexation kinetics between PVPh- $g$-PS and STVPy-25 also reflected in the fact that the formed micellar complexes possess a very loose structure; there also exists the probability that no well-defined core-shell nanostructure is even formed. We are currently unclear about the real structure of the loose aggregates from PVPh- $g$-PS/ STVPy-25.

From Figures 6 and 7, we successfully observed the kinetics of the formation of micellar complexes from PVPh- $g$-PS/ STVPy-49 and PVPh-g-PS/STVPy-74; the complexation occurs quite fast, which is complete within 1-2 s. Scattering intensities initially increase monotonically with time and then quickly stabilize at certain values. This is different from observations 


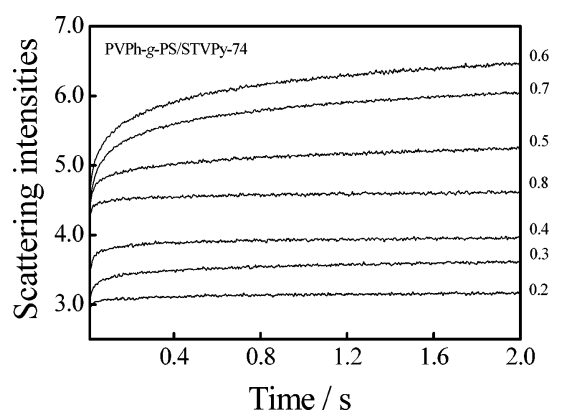

Figure 7. Typical time dependence of the scattering light intensities upon stopped-flow mixing PVPh- $g$-PS and STVPy-74 solutions in THF at different weight fractions of PVPh- $g$-PS; the total polymer concentration after mixing is $1.0 \times 10^{-4} \mathrm{~g} / \mathrm{mL}$.
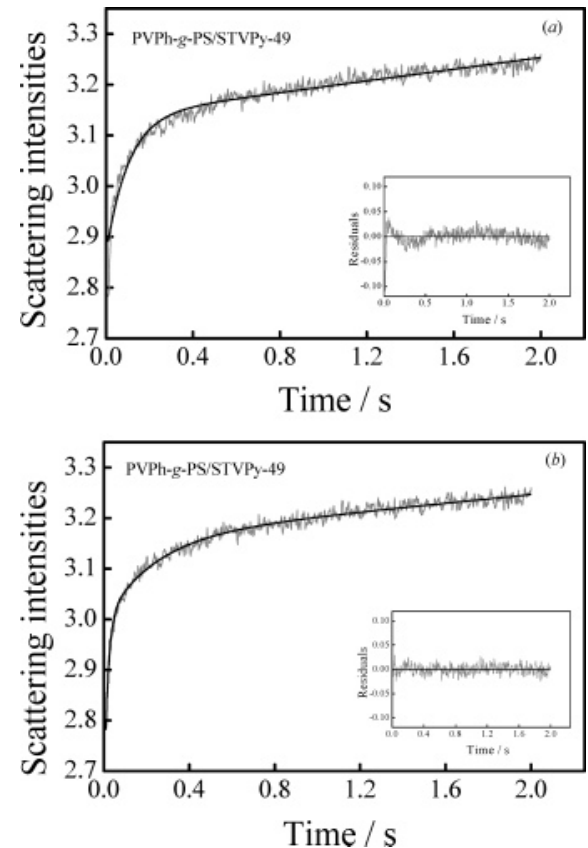

Figure 8. Typical time dependence of the scattering light intensities upon stopped-flow mixing PVPh- $g$-PS and STVPy-49 at a total polymer concentration of $1.0 \times 10^{-4} \mathrm{~g} / \mathrm{mL}$, where the weight fraction of PVPh$g$-PS is 0.5 . The upper (a) and lower (b) figures are fitted by singleand double-exponential functions, respectively. The inset is a plot of fitting residuals.

made by Stuart et al., ${ }^{15}$ which is the only report in the literature about kinetic studies of the formation of micellar complexes. They have studied the formation of micellar complexes via polyion complexation between PAA and PDMA- $b$-PGMA. Upon stopped-flow mixing, they observed the decrease of light scattering intensities with time; i.e., transient macroscopic phase separation occurs first, followed by rearrangement into the final stable micelles. In the absence of any salt and buffer, the process of the decrease of light scattering intensities takes about $\sim 1000$ s. In the current case, we do not observe the initial transient macroscopic phase transition. We are currently studying the kinetics of complexation between poly(ethylene oxide)- $b$-poly(trimethylaminoethyl methacrylate iodide) and poly(ethylene oxide)- $b$-poly(sodium styrenesulfonate), and we also observe the monotonic increase of scattering light intensities with time and subsequent stabilization at certain values.

The time dependence of the scattering light intensity $I_{t}$ can be converted to a normalized function, namely, $\left(I_{\infty}-I_{t}\right) / I_{\infty}$ vs $t$, where $I_{\infty}$ is the value of $I_{t}$ at an infinitely long time. Figure 8 show a typical plot of dynamic trace and the single- and double-exponential fitting results. The quality of the fit is assessed from the reduced $\chi^{2}$ error values, which is defined by

$$
\chi^{2}=\frac{1}{N} \sum_{i=1}^{N}\left(x_{i}-\bar{x}_{i}\right)^{2}
$$

where $N, x_{i}$, and $\bar{x}_{i}$ being the number of data points, the values of the experimental data, and the fitting data, respectively.

It was found that single-exponential function cannot fit the relaxation curve (Figure $8 \mathrm{a}$ ), especially for the first $0.1 \mathrm{~s}$, which is the most interesting to us because kinetics is the most accurate at initial stages. Empirically, we found that such a dynamic trace could be well fitted by a double-exponential function (Figure $8 b)$

$$
\left(I_{\infty}-I_{t}\right) / I_{\infty}=c_{1} \mathrm{e}^{-t / \tau_{1}}+c_{2} \mathrm{e}^{-t / \tau_{2}}
$$

where $c_{1}$ and $c_{2}$ are the normalized amplitudes $\left(c_{2}=1-c_{1}\right)$ and $\tau_{1}$ and $\tau_{2}$ are the characteristic relaxation time of two processes, $\tau_{1}<\tau_{2}$. The mean relaxation constant of the complexation process, $\tau_{\mathrm{f}}$, can be calculated as

$$
\tau_{\mathrm{f}}=c_{1} \tau_{1}+c_{2} \tau_{2}
$$

Both $\tau_{1}$ and $\tau_{2}$ have positive amplitudes. The $\chi^{2}$ error values improved $\sim 50 \%$ when double-exponential fitting is used instead of the single-exponential fitting. For the complexation between PVPh- $g$-PS and STVPy-49 at a weight fraction of $0.5, \tau_{1}$ and $\tau_{2}$ are $\sim 20$ and $280 \mathrm{~ms}$, respectively. The calculated $\tau_{\mathrm{f}}$ based on eq 4 is $120 \mathrm{~ms}$.

Morawetz et al. studied the kinetics of hydrogen-bonded interpolymer complexation between dansyl-labeled PAA and PEO. ${ }^{40}$ To avoid precipitation formation, they used excess of PEO. The kinetic traces are fitted with double-exponential functions, leading to two rate constants. However, they do not assign the two rate constants to the specific physical processes. Recently, Kiriy et al. ${ }^{57}$ have studied the interpolyelectrolyte complexation between high molecular weight poly(methacryloyloxyethyl dimethylbenzylammonium chloride) and short polyanion, sodium salt of poly(styrenesulfonate), by AFM on a single molecular basis. They propose that the complexation proceeds with initially fast forming nonequilibrium aggregates, followed by slow reactions (addition or/and substitution) into equilibrium polyelectrolyte complexes. On the basis of a similar mechanism, here we tentatively ascribe $\tau_{1}$ to the formation of the initial nonequilibrium aggregates and $\tau_{2}$ to the structural rearrangements into the final stable micellar complexes.

We then applied the same principle to the fitting of dynamic traces shown in Figures 6 and 7. For PVPh- $g$-PS/STVPy-49 (Figure 6), we found that the dynamic traces at PVPh- $g$-PS weight fraction $\sim 0.1,0.2$, and 0.9 can be adequately described by single-exponential fitting (Figure 6 , lower figure), and $\chi^{2}$ was not significantly improved by using double-exponential fitting, while double-exponential fitting was needed to fit all other dynamic curves shown in Figures 6 and 7.

Figure 9 shows the double-exponential function fitting results of the dynamic traces of PVPh- $g$-PS/STVPy-74 at various mixing ratios. $\tau_{1}$ is in the range $10-40 \mathrm{~ms}$, exhibiting a maximum at $\mathrm{PVPh}-g$-PS weight fraction $\sim 0.6$. Results shown in Figures 3 and 4 described earlier clearly tell us that at this composition the micellar complexes have the largest average density and scattering intensities; thus, it seems that $\tau_{1}$ is well associated with the stoichiometry of the micellar complexes. The complexation between PVPh- $g$-PS and STVPy proceeds stoichiometrically; the process associated with $\tau_{1}$, the formation 
of nonequilibrium aggregates, will be expected to be the slowest at the stoichiometric ratio. When the feed composition is far away from the stoichiometric ratio, this process will be faster due to the excess of one "reactant".

The change of $\tau_{2}$ with mixing ratios is different from that of $\tau_{1} . \tau_{2}$ is in the range of $0.15-0.3 \mathrm{~s} . \tau_{2}$ remains almost constant at $\sim 0.3 \mathrm{~s}$ when the weight fraction of $\mathrm{PVPh}-g$-PS is less than 0.6 ; above that, $\tau_{2}$ decreases with the weight fraction of PVPh$g$-PS. $\tau_{2}$ is associated with the structural rearrangement of the initially formed nonequilibrium aggregates into the final stable micellar complexes; this process will be accompanied by the addition or/and substitution of component polymer chains into the micellar complexes. ${ }^{55}$ At PVPh- $g$-PS weight fractions higher than 0.6 , the presence of excess of PVPh- $g$-PS may energetically favor the addition or/and substitution process due to less stretched PS chains at the shell of micellar complexes. ${ }^{58}$

\section{Conclusion}

We have synthesized PVPh- $g$-PS graft copolymer and STVPy random copolymers with different pyridyl contents, i.e., 25, 49, and $74 \mathrm{~mol} \%$. A combination of viscometry, LLS, and stoppedflow light scattering are employed to obtain the structural information on micellar complexes and the kinetics of the complexation between PVPh- $g$-PS and STVPy in THF. Hydrogen-bonding interactions between the PVPh backbone and STVPy lead to the formation of micellar complexes with the core consisting of insoluble complexes and the shell of wellsolvated PS grafts. We have successfully obtained the kinetics of the formation of micellar complexes by stopped-flow light scattering. The complexation between PVPh- $g$-PS and STVPy25 is so fast (complete within 1-2 ms) that no relaxation processes are observed at all. The formation of micellar complexes between PVPh-g-PS and STVPy-49 or STVPy-74 occurs within $\sim 1-2 \mathrm{~s}$. Typical relaxation traces at intermediate mixing ratios can be well-fitted with double-exponential functions. Two sequential steps in the assembly of micellar complexes can be resolved, which are assigned to formation of initial nonequilibrium aggregates $\left(\tau_{1}\right)$ and subsequent structural rearrangements into the final stable micellar complexes $\left(\tau_{2}\right)$ through addition or/and substitution of component polymer chains. The change of $\tau_{1}$ and $\tau_{2}$ with mixing ratios partially supports the above tentative assignments.

Acknowledgment. This work was supported by an Outstanding Youth Fund (50425310) and a key research grant (20534020) from the National Natural Scientific Foundation of China (NNSFC), the "Bai Ren" Project of the Chinese Academy of Sciences, and the Program for Changjiang Scholars and Innovative Research Team in University (PCSIRT).

\section{References and Notes}

(1) Colfen, H. Macromol. Rapid Commun. 2001, 22, 219-252.

(2) Riess, G. Prog. Polym. Sci. 2003, 28, 1107-1170.

(3) Alarcon, C. D. H.; Pennadam, S.; Alexander, C. Chem. Soc. Rev. 2005 , $34,276-285$.

(4) Rodriguez-Hernandez, J.; Checot, F.; Gnanou, Y.; Lecommandoux, S. Prog. Polym. Sci. 2005, 30, 691-724.

(5) Andre, X.; Zhang, M. F.; Muller, A. H. E. Macromol. Rapid Commun. 2005, 26, 558-563.

(6) Arotcarena, M.; Heise, B.; Ishaya, S.; Laschewsky, A. J. Am. Chem. Soc. 2002, 124, 3787-3793.

(7) Gohy, J. F.; Antoun, S.; Jerome, R. Macromolecules 2001, 34, 74357440.

(8) Rodriguez-Hernandez, J.; Lecommandoux, S. J. Am. Chem. Soc. 2005 , 127, 2026-2027.

(9) Butun, V.; Billingham, N. C.; Armes, S. P. J. Am. Chem. Soc. 1998, 120, 11818-11819.

(10) Liu, S. Y.; Armes, S. P. Angew. Chem., Int. Ed. 2002, 41, 14131416.

(11) Liu, S. Y.; Armes, S. P. Langmuir 2003, 19, 4432-4438.

(12) Liu, S. Y.; Billingham, N. C.; Armes, S. P. Angew. Chem., Int. Ed. 2001, 40, 2328

(13) Liu, S. Y.; Weaver, J. V. M.; Tang, Y. Q.; Billingham, N. C.; Armes, S. P.; Tribe, K. Macromolecules 2002, 35, 6121-6131.

(14) Evans, D. F.; Wennerstrom, H. The Colloidal Domain: Where Physics, Chemistry, Biology, and Technology Meet; VCH Publishers: New York, 1994.

(15) Stuart, M. A. C.; Besseling, N. A. M.; Fokkink, R. G. Langmuir 1998, 14, 6846-6849.

(16) van der Burgh, S.; de Keizer, A.; Stuart, M. A. C. Langmuir 2004, 20, 1073-1084.

(17) Harada, A.; Kataoka, K. Macromolecules 1995, 28, 5294-5299.

(18) Harada, A.; Kataoka, K. Macromolecules 1998, 31, 288-294.

(19) Harada, A.; Kataoka, K. Langmuir 1999, 15, 4208-4212.

(20) Kataoka, K.; Togawa, H.; Harada, A.; Yasugi, K.; Matsumoto, T.; Katayose, S. Macromolecules 1996, 29, 8556-8557.

(21) Yuan, X. F.; Harada, A.; Yamasaki, Y.; Kataoka, K. Langmuir 2005 $21,2668-2674$

(22) Yuan, X. F.; Yamasaki, Y.; Harada, A.; Kataoka, K. Polymer 2005, 46, 7749-7758.

(23) Kabanov, A. V.; Vinogradov, S. V.; Suzdaltseva, Y. G.; Alakhov, V. Y. Bioconjugate Chem. 1995, 6, 639-643.

(24) Kabanov, A. V.; Bronich, T. K.; Kabanov, V. A.; Yu, K.; Eisenberg, A. Macromolecules 1996, 29, 6797-6802. 
(36) Hou, S. J.; Man, K. Y. K.; Chan, W. K. Langmuir 2003, 19, 24852490

(37) Zhao, H. Y.; Douglas, E. P.; Harrison, B. S.; Schanze, K. S. Langmuir 2001, 17, 8428-8433

(38) Ishizu, K.; Minematsu, S.; Fukutomi, T. J. Appl. Polym. Sci. 1991, 43, 2107-2114.

(39) Chen, H. L.; Morawetz, H. Macromolecules 1982, 15, 1445-1447.

(40) Bednar, B.; Morawetz, H.; Shafer, J. A. Macromolecules 1984, 17, $1634-1636$.

(41) Oupicky, D.; Reschel, T.; Konak, C.; Oupicka, L. Macromolecules 2003, 36, 6863-6872.

(42) Braun, C. S.; Fisher, M. T.; Tomalia, D. A.; Koe, G. S.; Middaugh, C. R. Biophys. J. 2005, 88 .

(43) Johnson, K. A. Kinetic Analysis of Macromolecules: A Practical Approach; Oxford University Press: New York, 2003.

(44) Wu, K.; Shi, L. Q.; Zhang, W. Q.; An, Y. L.; Zhu, X. X.; Zhang, X.; Li, Z. Y. Soft Matter 2005, 1, 455-459.

(45) Zhu, Z. Y.; Gonzalez, Y. I.; Xu, H. X.; Kaler, E. W.; Liu, S. Y. Langmuir 2006, 22, 949-955.

(46) Xu, J.; Zhu, Z. Y.; Luo, S. Z.; Wu, C.; Liu, S. Y. Phys. Rev. Lett. 2006, 96, 027802.

(47) Dai, J.; Goh, S. H.; Lee, S. Y.; Siow, K. S. Polym. J. 1994, 26, 905911.
(48) Xiang, M. L.; Jiang, M.; Zhang, Y. B.; Wu, C.; Feng, L. X. Macromolecules 1997, 30, 2313-2319.

(49) Zhang, Y. B.; Xiang, M. L.; Jiang, M.; Wu, C. Macromolecules 1997, 30, 6084-6089.

(50) Liu, S. Y.; Weng, L. T.; Chan, C. M.; Li, L.; Ho, N. K.; Jiang, M. Surf. Interface Anal. 2001, 31, 745-753.

(51) Wu, C.; Xia, K. Q. Rev. Sci. Instrum. 1994, 65, 587-590.

(52) Zhu, Z. Y.; Armes, S. P.; Liu, S. Y. Macromolecules 2005, 38, 98039812.

(53) Jiang, M.; Li, M.; Xiang, M. L.; Zhou, H. Adv. Polym. Sci. 1999, 146, 121-196.

(54) Teraoka, I. Polymer Solutions: An Introduction to Physical Properties; John Wiley \& Sons: New York, 2002.

(55) Xu, R. L.; Winnik, M. A.; Hallett, F. R.; Riess, G.; Croucher, M. D. Macromolecules 1991, 24, 87-93.

(56) Bednar, B.; Edwards, K.; Almgren, M.; Tormod, S.; Tuzar, Z. Makromol. Chem., Rapid Commun. 1988, 9, 785-790.

(57) Kiriy, A.; Yu, J.; Stamm, M. Langmuir 2006, 22, 1800-1803.

(58) Bakeev, K. N.; Izumrudov, V. A.; Kuchanov, S. I.; Zezin, A. B.; Kabanov, V. A. Macromolecules 1992, 25, 4249-4254. MA060581Y 